\title{
The Pharmacological Activities of Glycyrrhizinic Acid ("Glycyrrhizin") and Glycyrrhetinic Acid
}

\author{
Cedric Stephan Graebin
}

\section{Contents}

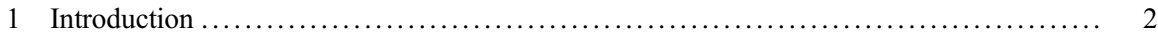

2 Pharmacological Activities of Glycyrrizinic Acid ("Glycyrrhizin") and Related

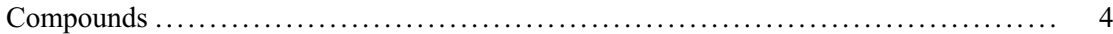

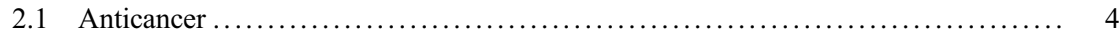

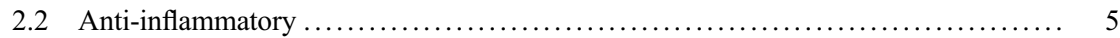

2.3 Hepatoprotective Effects ............................................ 6

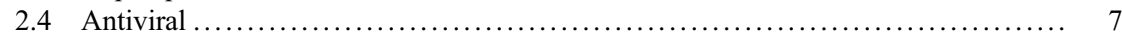

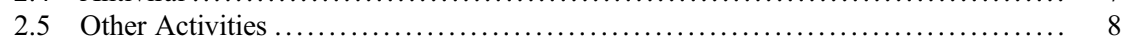

3 Pharmacological Activities of Glycyrrhetinic Acid (2) and Related Compounds ......... 9

3.1 Anticancer Activity .................................................... 9

3.2 Hepatoprotective Activity .......................................... 10

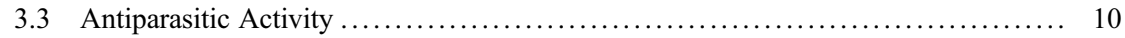

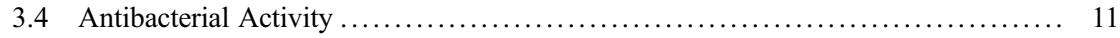

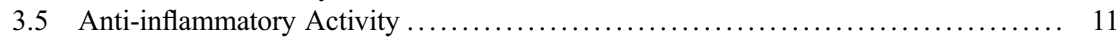

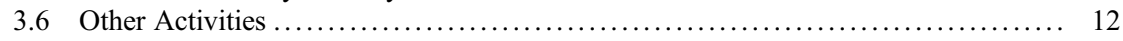

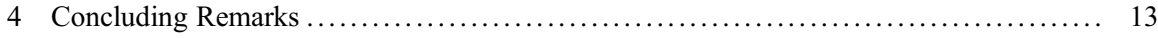

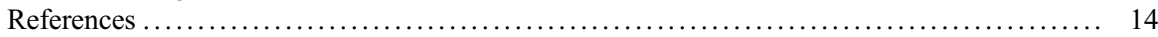

\section{Abstract}

Glycyrrhizin or, more correctly, Glycyrrhizinic acid is a triterpenoid saponin obtained from the root and rhizome extracts of Licorice (Glycyrrhiza glabra), being commonly used as a sweetener, being reported as - at least - 30 times sweeter than sucrose. This natural product, along with its aglycone glycyrrhetinic

C.S. Graebin $(\bowtie)$

Chemistry Department, Federal Rural University of Rio de Janeiro, Seropédica, RJ, Brazil e-mail: cedric@ufrrj.br 
acid, is known in the literature for its several pharmacological and biological activities. This chapter summarizes the activities reported in the literature for the saponin and its aglycone since 2010 .

\section{Keywords}

Glycyrrhizinic acid • Glycyrrhizin • Glycyrrhetinic acid • Glycyrrhiza glabra • Liquorice

\section{Introduction}

Glycyrrhizinic acid (1, Fig. 1), also known as Glycyrrhizin [1, 2], is a triterpenoid saponin obtained from the root and rhizome extracts of Liquorice (or "licorice" in US English) (Glycyrrhiza glabra). Normally, the dried licorice root extract may contain around $4-25 \%$ of the saponin, along with other compounds such as polyphenols, saponins, triterpenes, etc. One of the triterpenes found in the extract is the triterpenic aglycone, glycyrrhetinic acid, or "enoxolone" (2). Further purification using several extraction techniques gives usually the monoammonium glycyrrhetinate salt as the purified product [2].

The World Health Organization (WHO) report on Glycyrrhizin recommends that the compound must be named "Glycyrrhizinic acid." According to the report, "Glycyrrhizin" is the name given, more correctly, to the Licorice extract and not the saponin itself, and "Glycyrrhizin" and "Glycyrrhizinic acid" should not be used as synonyms [1], although this is common practice in the literature. This chapter will follow this recommendation and use the second term as the saponin name.

Both the saponin (as a carboxylic acid) and its monoammonium salt are commonly employed as sweeteners, being reported as 30-50 [3] or 33-200 [2] times

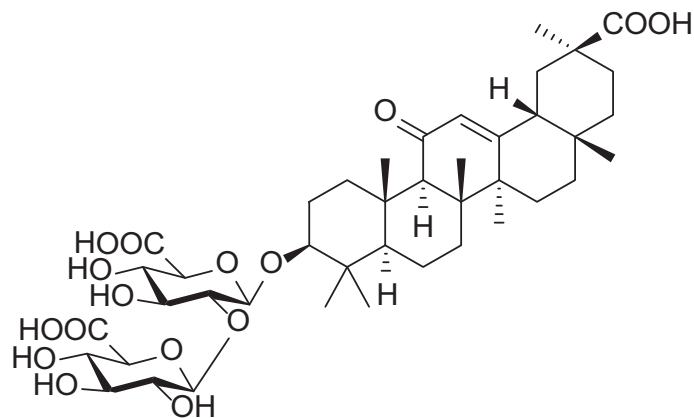

Glycyrrhizinic acid "Glycyrrhizin" 1

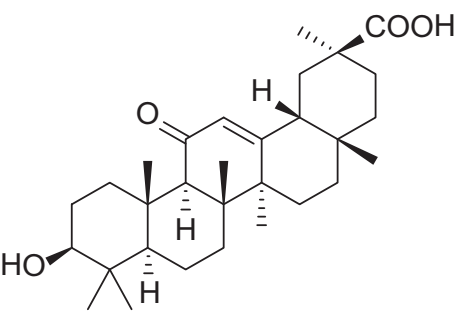

Glycyrrhetinic acid or $18 \beta$-Glycyrrhetinic acid

2

Fig. 1 Chemical structures of Glycyrrhizinic (1) and Glycyrrhetinic acid (2) 


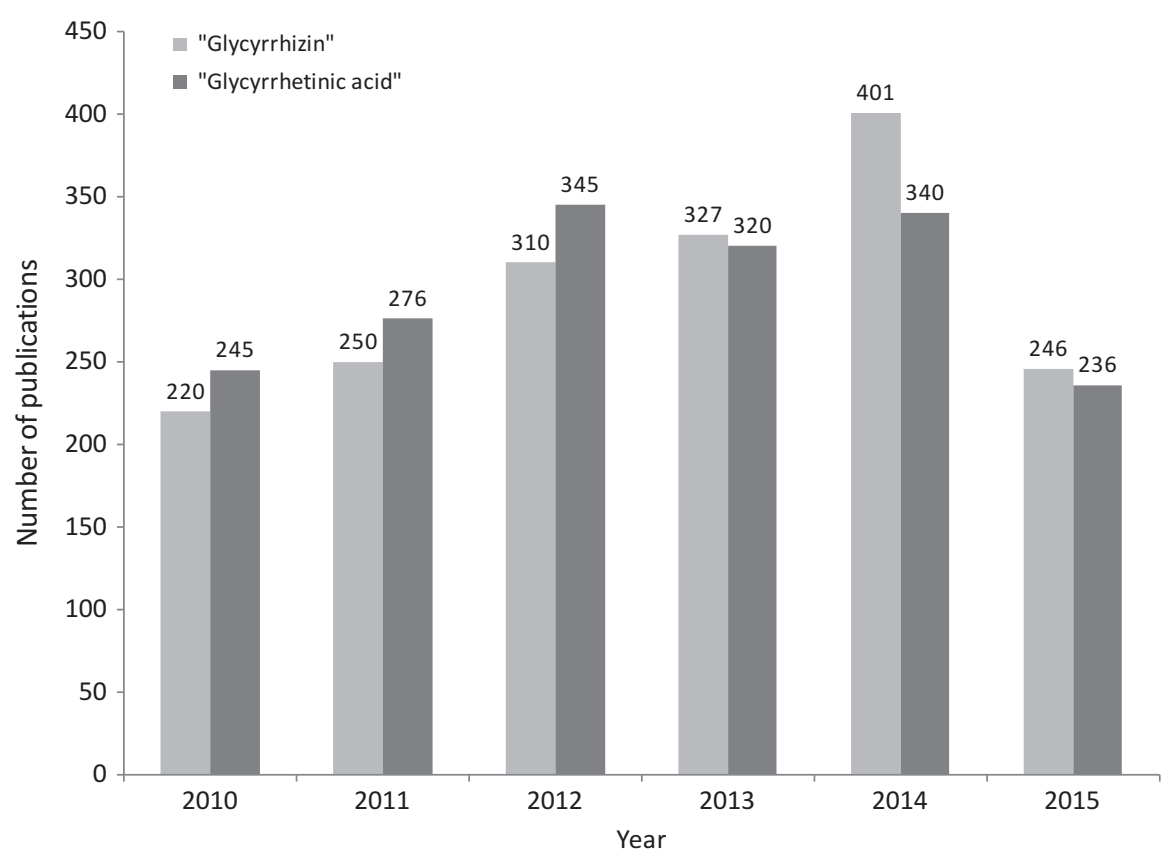

Fig. 2. Publications mentioning the terms "Glycyrrhizin" or "Glycyrrhetinic acid" in its titles and/or abstract in SciFinder database (year period: 2010-2015)

sweeter than sucrose. The saponin is also widely employed in several traditional Chinese, Tibetan, and Indian medicinal preparations [3]. "Stronger Neo-Minophagen C," a glycyrrhetinic acid-containing i.v. preparation, is employed in the treatment of chronic hepatic diseases, being marketed in Japan, China, Korea, Taiwan, Indonesia, India, and Mongolia [4].

The intent of this chapter is to report the wide range of pharmacological activities reported in the literature for the saponin (1) and its aglycone (2). The inclusion of the aglycone in this chapter is justified due to the fact that the saponin has no oral bioavailability, being absorbed as glycyrrhetinic acid (2) after hydrolysis of its carbohydrate moiety by intestine bacteria [5]. Since there are several reports published regarding those compounds until $c a .2010$ [3, 6, 7], the time period covered by this chapter is from 2010 onwards.

Using SciFinder scientific literature search service [8], several articles mentioning "Glycyrrhizin" or "Glycyrrhetinic acid" in its titles and/or abstracts were found (Fig. 2). As mentioned earlier, using "Glycyrrhizinic acid" as search term gives a reduced number of articles as response. Due to the enormous number of publications in the selected period, it was decided to focus this chapter in the publications reporting pharmacological activities to the saponin, its aglycone, and some closely related compounds. Several Japanese, Korean, and (mostly) Chinese patents found in the bibliographic research mentioning $\mathbf{1}$ are related to its use as a sweetener/ 


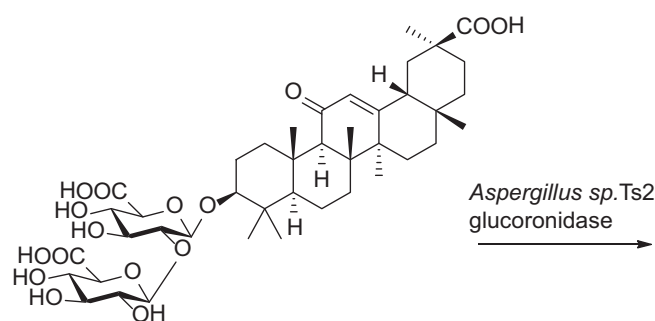

1

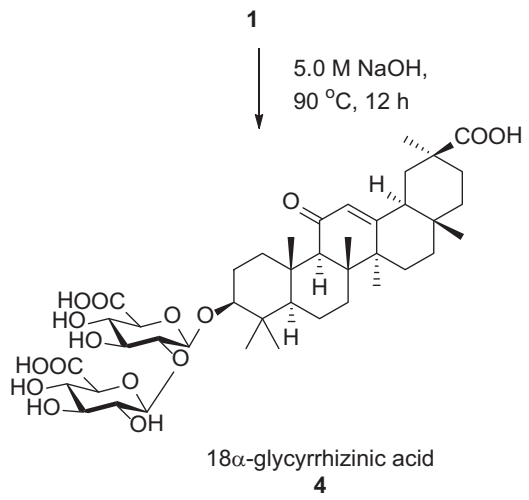

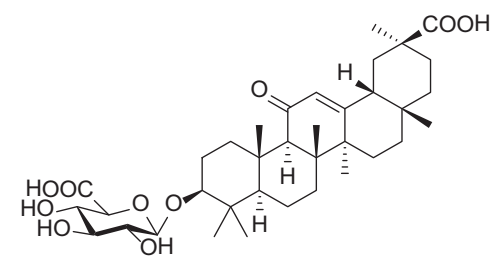

3

$5.0 \mathrm{M} \mathrm{NaOH}$, $90^{\circ} \mathrm{C}, 12 \mathrm{~h}$

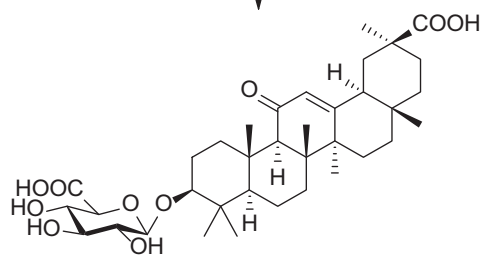

$18 \alpha$-glycyrrhizinic acid mono glucoronide 5

Scheme 1 Synthesis of $18 \alpha$-epimers of Glycyrrhizinic acid

flavoring agent in herbal/drug preparations and, therefore, were left out of this review.

\section{Pharmacological Activities of Glycyrrizinic Acid ("Glycyrrhizin") and Related Compounds}

\subsection{Anticancer}

Several findings were reported in the last few years regarding the anticancer properties of Glycyrrhizinic acid. Wang et al. reported the in vitro activity of $\mathbf{1}$ against gastric cancer cell line BCG-823. The saponin was able to prevent cell proliferation, adhesion, and migration at a $40 \mu \mathrm{M}$ dose and they observed also that the expression of $\beta$-catenin, Bcl-2, CyclinD1, and survivin was significantly decreased [9].

In order to evaluate the carbohydrate moiety importance in the anticancer properties of 1, Yang et al. synthesized two monoglucuronides from $\mathbf{1}$ by means of a glucuronidase from Aspergillus sp. (Scheme 1). The authors reported that 18$\alpha$-monoglucuronide (5) obtained was more effective against HepG2, HeLa, and A549 cancer cell lines ( $\mathrm{IC}_{50}$ values: $6.67,7.43$, and $15.76 \mu \mathrm{M}$, respectively) than $18 \beta$-monoglucuronide (3), 1, and 18 $\alpha$-glycyrrhizinic acid (4). The monoglucuronide 3 was also the best Epidermal Growth Factor Receptor (EGFR) inhibitor, with a $\mathrm{IC}_{50}$ 
of $0.028 \mu \mathrm{M}$. Molecular docking simulations indicate that 3 also is able to bind with EGFR, suggesting that the anticancer properties of this glucuronide are related to EGFR inhibition [10].

Huang et al., in order to clarify the mechanism of the anticancer activity of 1 against lung adenocarcinoma A549 cell lines, evaluated the expression of several enzymes in an in vitro model and found that the anticancer activity of $\mathbf{1}$ against this cancer cell line can be, according to the authors, related to the inhibition of Thromboxane Synthase by $\mathbf{1}$. The same results were also found in an in vivo mouse model using a western blot assay [11].

The inhibition of High-Mobility Group Box 1 (HMGB1), a protein that acts outside the cellular milieu as a proinflammatory cytokine, can also be related to the anticancer properties of $\mathbf{1}$. Smolarczyk et al. reported that, when employing in an in vivo tumor model, the use of $\mathbf{1}$ inhibited cell growth and proliferation in mice. The authors concluded that the cell growth inhibition was probably due to the inhibition of HMGB1 by $\mathbf{1}$ in the extracellular media [12].

\subsection{Anti-inflammatory}

Wang et al. determined that $\mathbf{1}$ was able to attenuate levels of Tumor Necrosis Factor$\alpha(\mathrm{TNF}-\alpha)$, interleukin $1 \beta$ (IL-1 $\beta$ ), and activation of the HMGB1/NFkB (Nuclear factor-kB) signaling pathway in the hippocampus of neonatal rats after exposure to isoflurane (a general anesthetic drug). Moreover, treatment with $\mathbf{1}$ also prevented the deficits in spatial memory related to isoflurane [13].

Using a pancreatic trauma model in male Wistar rats, Xiang et al. reported that treatment with 1, 15 min after inducing the trauma, led to lower serum levels of HMGB-1, TNF- $\alpha$, and IL- 6 when compared to the control group. The treatment with the saponin also increased the seven-day survival rate of the animal subjects in this experimental model.

Zhang et al. reported that $\mathbf{1}$ has a neuroprotective effect in the postischemic brain in mice through the HMGB1-TLR4-IL-17A pathway [14].

Glycyrrhizinic acid (1) and Licorice flavonoids, tested in vitro in separate groups, were found by Zhao et al. to modulate the secretion of several cytokines by macrophages (RAW 264.7 cells) induced by lipopolysaccharides (LPS) [15]. Fu et al. reported that 1 presented its anti-inflammatory activity in LPS-stimulated RAW 264.7 cells by disrupting lipid rafts and inhibiting Toll-like receptor-4 (TLR-4) translocation to those rafts [16].

Wang et al. reported that $\mathbf{1}$ was able to reduce xylene, carrageenan, and acetic acid-induced rat paw edema and the nociceptions induced by formalin and acetic acid, but it had no effects in the hot plate test. The authors also observed the downregulation of levels of TNF- $\alpha$, IL-6, inducible Nitric Oxide Synthase (iNOS), and Cyclooxigenase-2 (COX-2) [17].

Investigating the effects of $\mathbf{1}$ in the subarachnoid hemorrhagic (SAH) rat model, Chang et al. reported that the saponin exerted anti-inflammatory effects reducing $\mathrm{SAH}$-induced vasospasm, mostly through the attenuation of peroxisome 
proliferator-activating receptor gamma (PPAR- $\gamma$ ) [18]. The same research group reported that, in the same $\mathrm{SAH}$ model, treatment with $\mathbf{1}$ was also able to attenuate the ultrashort time expression of Toll-like receptors (TLR) 2 and 4 [19].

The saponin was also subject of some clinical trials regarding its antiinflammatory activity. Xiao et al. conducted a randomized trial with 39 children presenting Henoch-Schonlein purpura (HSP) in order to verify the functions of regulatory T-cells (Treg) and Th17 cells in the peripheral blood of those patients. The group treated with 1 had significantly different Interleukin-17 (IL-17) serum levels after the treatment but no difference in Transforming Growth Factor-beta (TGF- $\beta$ ) and Interleukin-10 (IL-10) serum levels after the treatment. The authors concluded that 1 was able to reduce Th17 function, without noticeable effects in Treg function [20].

Glycyrrhizinic acid was also trialed in patients with chronic urticaria. Eighty four patients with the disease were randomized in two groups: one with 1 (50 mg oral tablets, three times per day) and the other with levocetirizine (an antiallergic drug). Both compounds were administered during a four-week period. The authors concluded that 1 was superior to levocetirizine and could be used to treat chronic urticaria [21]. It is worth noticing that $\mathbf{1}$ has no oral bioavailability, being hydrolyzed to form glycyrrhetinic acid (2) before being absorbed [1]. Therefore, the observed pharmacological activity is probably due to the known anti-inflammatory activity of $\mathbf{2}$ (see section 3 of this chapter) rather than $\mathbf{1}$.

\subsection{Hepatoprotective Effects}

Magnesium isoglycyrrhizinate, the magnesium salt of $18 \alpha$-glycyrrhizinic acid (4), was found by Luo et al. to be an effective treatment against hepatitis $E$ with severe jaundice during a clinical trial with 78 patients. The patients received an intravenous injection of $150 \mathrm{mg}$ of the magnesium once a day during 6 weeks [22].

Zhang et al. conducted a clinical trial with 84 patients with digestive tract cancer in order to verify the hepatoprotective activity of $\mathbf{1}$ during standard cancer chemotherapy. The authors observed that the group undergoing chemotherapy and treated with the saponin (160 mg i.v. once a day) presented significantly lower liver transaminase levels and increased levels of neutrophile, granulocytes, and white blood cells when compared with the control group (standard chemotherapy only) [23].

Also while investigating the hepatoprotective activity of $\mathbf{1}$, Hsiang et al. investigated the gene expression of HepG2 cells treated in vitro independently with $\mathbf{1}$, silymarin, and ursodeoxycholic acid, natural products also known by their hepatoprotective properties. The authors concluded that the compounds inhibited NF- $\kappa \mathrm{B}$ activities in a dose-dependent manner and that those compounds regulated the expression of genes related to oxidative stress and apoptosis in those cells [24].

Meng et al. conducted a meta-analysis in 12 randomized clinical trials regarding the hepatoprotective activity of $\mathbf{1}$ in patients with alcoholic liver disease. Their analysis concluded that in those trials the levels of alanine and aspartate 
aminotransferases, as well as $\gamma$-glutaryl transpeptidase were lower than the control groups after treatment with the saponin [25].

During an investigation with patients with chronic hepatitis B, Yin et al. found that a three-week treatment with $\mathbf{1}$ did not reduce the expressions of inflammatory cytokine IL-17 and antinuclear antibodies (ANA) in the treated patients when compared to the control group [26].

Treating chronic hepatitis B with either Magnesium isoglicyrrhizinate or $\mathbf{1}$ was compared in a clinical trial reported by Cai et al. The trial involved 64 patients which were divided in two groups (one group being treated intravenously with the Magnesium salt and the other with the saponin, also i.v., once a day for 4 weeks). In both cases liver function was improved in the patients, but the authors found no statistical difference between both treatments [27].

Ding et al. investigated the effect of $18 \alpha$-glycyrrhizinic acid (4) in rats with carbon tetrachloride $\left(\mathrm{CCl}_{4}\right)$-induced liver fibrosis. It was found that treatment with 4 increased the activity of enzymes glutathione peroxidase and superoxide dismutase, which reduced lipid peroxidation and the levels of malonodialdehyde and hydroxynonenal in the liver, protecting it from damage caused by the aldehydes [28].

Wang et al. found that the treatment of HepG2 cells with $\mathbf{1}$ caused the increase in both CYP3A mRNA and protein levels. The CYP3A gene encodes monooxygenases responsible for drug metabolism and steroid biosynthesis [29]. The authors also found that the induction of CYP3A was mediated through the activation of Pregnane $\mathrm{X}$ receptor (PXR), resulting in the induction of CYP3A11 expression and CYP7A1 inhibition [30].

The hepatoprotective effect of $\mathbf{1}$ was also studied using HL-7702 (normal human liver cell line) through acetaminophen-induced damage. According to the report by Chen et al., 1, liquirtin, and isoliquirtigenin (two polyphenols also found in the Liquorice root extract) were effective as hepatoprotecting agents against acetaminophen-induced damage [31].

Gwak et al., investigating the role of HMGB-1 in hepatocyte apoptosis, found that 1, known as a HMGB-1 inhibitor, was able to prevent HMGB-1-induced apoptosis, cytochrome $\mathrm{C}$ release, and p38 activation in Huh-BAT (human hepatocellular carcinoma transfected with bile acid transporter) cell line [32].

\subsection{Antiviral}

Glycyrrhizinic acid (1) is known in the literature as an antiviral agent against clinically relevant viruses, such as HIV and the SARS-Coronavirus [3, 6]. Duan et al. reported that $\mathbf{1}$ is active against porcine reproductory and respiratory syndrome virus (PRRSV), relevant in veterinary medicine and in the swine industry. The saponin was able to inhibit mainly the penetration stage of the virus cycle [33].

Investigating the known anti-HIV activity of the saponin, $\mathrm{Li}$ et al. found that $\mathbf{1}$, among 27 compounds isolated from Liquorice extract, has the high binding constant for $\mathrm{R} 15 \mathrm{~K}$, the conserved core sequence in the V3 loop region of gp120. This region, 


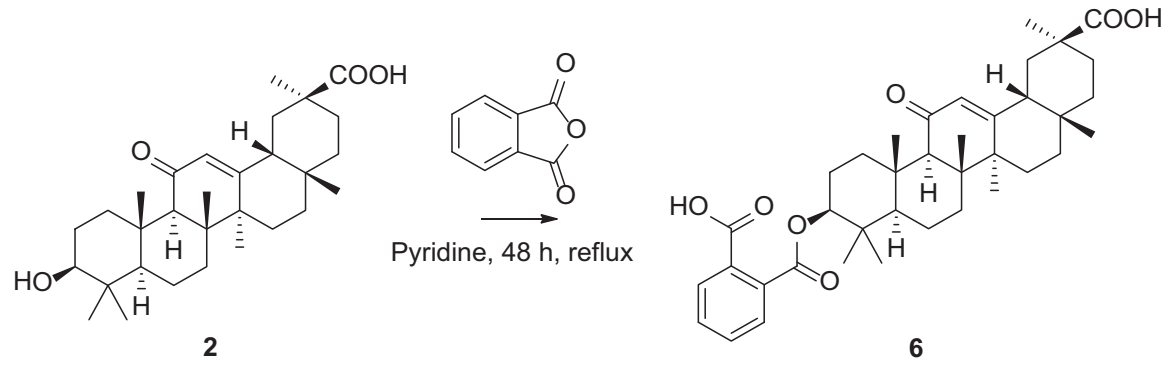

Scheme 2 Synthesis of glycyrrhetinic acid 3-O-hemiphtalate

according to the authors, is where the binding of glycophospholipids occurs, leading to the virus entry in the host cell, which can indicate that the saponin is having antiHIV activity by inhibiting the virus entry stage [34].

Using a Hepatitis C virus (HCV)-infected Huh7 cell model, Matsumoto et al. reported that 1 inhibits the release of HCV particles. Suppression of viral entry and reproduction stages were not observed [35]. Ashfaq et al., also investigating the anti-HCV activity of the saponin, reported that treatment of HCV-infected hepatic cells led to the suppression of HCV 3 a core gene both at mRNA and protein levels. Coadministration of $\mathbf{1}$ with interferon alpha-3a caused a synergistic effect [36].

\subsection{Other Activities}

Xiao and Zhou reported that 1 reduced the Human neutrophil elastase-induced Mucin 5 AC (MUC5AC) overproduction in human bronchial epithelial cell culture (16HBE) [37].

The interaction of 1 with human hemoglobin $(\mathrm{Hb})$ was studied by Sil et al. The authors reported that the interaction between the saponin and the protein reduces $\mathrm{Hb}$-mediated oxidative damage without affecting the oxygen binding properties of $\mathrm{Hb}[38]$.

The antithrombin activity of $\mathbf{1}$ was already known since 1997 [39]. Glycyrrhizinic acid was found to be a weak in vitro and in vivo thrombin inhibitor but, unlike other antithrombin agents, the saponin acts as an allosteric inhibitor [40]. Glycyrrhetinic acid (2), however, has no thrombin inhibiting activity, indicating that the carbohydrate moiety of the saponin is important to the allosteric site interaction. With these results in mind, Paula et al. synthesized some derivatives aiming to simplify the carbohydrate scaffold. From all the synthesized compounds, the hemiphtalate 6 (Scheme 2) presented better in vitro antithrombin activity against thrombin and increased the thrombin time, indicating that the carboxylic acid moiety present in the saponin (and in the phthalate) is important to the recognition at the allosteric site [41]. 
Investigating the free-radical scavenging abilities of 1, Imai et al. reported that 1 and glycyrrhetinic acid (2) were able to scavenge 1,1-diphenyl-2-picrylhydrazyl (DPPH) radicals but not hydroxyl and superoxide anion radicals [42].

\section{Pharmacological Activities of Glycyrrhetinic Acid (2) and Related Compounds}

As mentioned before, $\mathbf{2}$ is the compound absorbed in the human blood (due to the hydrolysis of $\mathbf{1}$ by bacterial metabolism) after oral administration of the saponin [1]. It was decided, therefore, to report the new pharmacological properties described for the aglycone in the literature below.

\subsection{Anticancer Activity}

There are several reports regarding the design, synthesis, and pharmacological evaluation of glycyrrhetinic acid derivatives towards compounds with improved anticancer properties. Since this chapter is focused mainly in the pharmacological properties of $\mathbf{1}$ and $\mathbf{2}$ itself (along with some closely related derivatives), only reports regarding the anticancer activity of $\mathbf{2}$ itself will be detailed below. Readers interested in more in-depth articles regarding chemical modifications in the aglycone structure are recommended to the review article by Czuk [43] as well as other review articles previously mentioned in the chapter $[3,6]$.

Kim et al. reported that $\mathbf{2}$ presented in vitro cytotoxic activity against A549 (human lung adenocarcinoma) cell line and also was able to reduce in vivo tumors induced by the same cell culture injected in Balb/c nu/nu mice [44].

Employing r/m HM-SFCE-1 cells (highly metastatic ras/myc-transformed serumfree mouse embryo), Yamaguchi et al. found that the cytotoxic effect of $\mathbf{2}$ in this model was due to the downregulation of glutathione, disrupting the redox balance in those cells [45].

$\mathrm{Xu}$ et al., using the human myeloma cell line (U266) reported that $\mathbf{2}$ induced in vitro apoptosis in this cell line by downregulating the survivin gene expression and arresting the cells in $\mathrm{G}_{0} / \mathrm{G}_{1}$ phase [46].

Aiming to study the potential use of $\mathbf{2}$ as a chemoprotective agent, Kowsalya et al. induced buccal pouch carcinogenesis in hamsters using 7,12-dimethylbenz(a) anthracene (DMBA) as a tumor initiator. Oral administration of $2(45 \mathrm{mg} / \mathrm{kg})$ along with DMBA prevented tumor formation in this animal model [47].

Charma et al. reported that the apoptotic effect of $\mathbf{2}$ in human breast cancer cell line MCF-7 was due to the activation of caspase-9 and modulation of the Protein Kinase B/Forkhead box O 3a transcription factor (Akt/FOXO3a) pathway [48].

Working with hepatic stellate cells (HSC), cells with immunosuppressive capabilities and related to the development of hepatocellular carcinomas, Kuang et al. found that $\mathbf{2}$ is able to reduce in vitro HSC-mediated immunosuppression by 
reducing T-cell apoptosis and regulatory $\mathrm{T}$ (Treg) cell expression, which in turn lead to an increase in T-cell activity against hepatocellular carcinoma cells [49].

Xie et al. reported that $\mathbf{2}$ inhibited the epidermal growth factor (EGF)-induced proliferation of $\mathrm{HaCaT}$ cells (human keratinocyte cell line), likely by suppressing the extracellular signal-regulated kinase (ERK1/2) signaling pathway [50].

An in vitro study with prostate cancer cell line LNCaP conducted by $\mathrm{Li}$ et al. indicates that the activity of $\mathbf{2}$ in those cells is probably due to the suppression of 17 $\beta$-hydroxysteroid dehydrogenase type III (17 $\beta$-HSD3) mRNA expression via activation of eukaryotic initiation factor $2 \alpha(\operatorname{eIF} 2 \alpha)$ [51].

Wang et al. investigated the cytotoxic effect of $\mathbf{2}$ in pituitary adenoma cancer cell lines MMQ and GH3. The authors reported that the triterpene induced apoptosis in those cells by activating mitochondria-mediated reactive oxygen species (ROS)/ mitogen-activated protein kinase (MAPK) pathways [52].

Huang et al. reported that $\mathbf{2}$ is able to suppress proliferation of non-small cell lung cancer (NSCLC) lines cells A549 and NCI-H460 through inhibition of thromboxane synthase and activation of ERK/CREB signaling [53].

Investigating the effect of $\mathbf{2}$ in the metastatic and cell-invading ability of several cancer cell lines, Jayasooriya et al. found that $\mathbf{2}$ is capable of downregulating matrix metalloproteinase-9 (MMP-9) and vascular endothelial growth factor (VEGF) via inhibition of phosphatidyl-inositol 3 kinase (PI3K)/Akt-dependent NF- $\mathrm{BB}$ activity [54].

\subsection{Hepatoprotective Activity}

As reported with Glycyrrhizinic acid (1), 2 also presents hepatoprotective activity. Mahmoud et al. observed that $\mathbf{2}$ was able to counteract cyclophosphamide-induced hepatotoxicity in rats via activation of nuclear factor-erythroid 2 (NF-E2) related factor 2 (Nrf2) and PPAR- $\gamma$ [55].

The factor Nrf2 is also involved in the chemoprotective mechanism of $\mathbf{2}$ against carbon tetrachloride-induced liver fibrosis in mice. Chen et al. reported that the triterpene is able to upregulate $\mathrm{Nrf} 2$ and increase the activity of antioxidant enzymes in the liver [56].

Glycyrrhetinic acid is also a chemoprotective agent against 2-acetylaminofluorene-induced hepatotoxicity in Wistar rats via attenuation of oxidative stress, inflammation, and hyperproliferation, according to Hasan et al [57].

\subsection{Antiparasitic Activity}

Investigating the antileishmanial activity of $\mathbf{2}$ in experimental visceral leishmaniasis models, Gupta et al. reported that this activity occurs through nitric oxide (NO) upregulation, proinflammatory cytokine expression and NF- $\mathrm{\kappa B}$ activation through p38 kinase [58]. The same research group later reported that this activity also depends on phosphatase-dependent modulation of cellular MAP kinases [59]. 
Fig. 3. Chemical structure of $18 \alpha$-glycyrrhetinic acid (7)

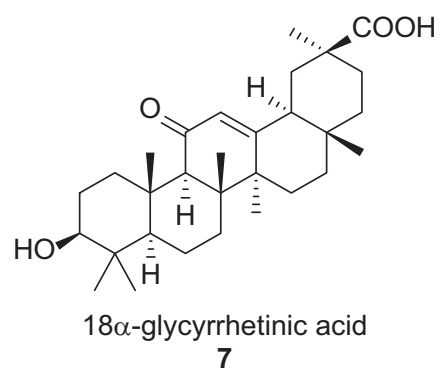

Kalani et al., in an effort to find new affordable antimalarial agents, reported that 2 has in vitro anti- $P$. falciparum activity, with $\mathrm{IC}_{50}=1.69 \mu \mathrm{g} / \mathrm{mL}$, and in vivo activity during an 8-day course treatment [60]. The same research group found that $\mathbf{2}$ is also an antifilarial agent, being active in vitro against microfilariae but not against adult worms. Some 2-related amides synthesized by the group were able to affect both microfilariae and adult worms [61].

\subsection{Antibacterial Activity}

Long et al. reported that $\mathbf{2}$ is active against Methicilin-resistant Staphylococcus aureus (MRSA), a major source of complicated infections in hospital environments. According to the authors, 2 presented in vitro bactericidal activity at $0.223 \mu \mathrm{M}$ and, in sublethal concentrations, it is able to reduce the expression of genes related to the S. aureus virulence, such as saeR and hla [62].

Investigating the effect of $18 \alpha$-glycyrrhetinic acid (7, Fig. 3) on the periodontal pathogen Porphyromonas gingivalis, Kim et al. reported that 7 reduces the bacterial LPS-induced permeability by suppressing repressing NF- $\mathrm{KB}-\mathrm{dependent}$ endothelial IL-8 production [63].

\subsection{Anti-inflammatory Activity}

Glycyrrhetinic acid (2) is also known for its anti-inflammatory activity, acting as a $11 \beta$-hydroxysteroid dehydrogenase inhibitor. This enzyme is responsible for the conversion of cortisol (8, Fig. 4) to cortisone and, as a result, $\mathbf{2}$ is an indirect glucocorticoid anti-inflammatory agent by extending cortisol half-life $[1,6]$. Carbenexolone (9), the hemisuccinate ester of $\mathbf{2}$, is used as an antiinflammatory drug in United Kingdom $[3,6]$. The chemical structures of $\mathbf{2}, \mathbf{8}$, and 9 are shown in Fig. 4.

Since there are several reports regarding the design, synthesis, and pharmacological evaluation of glycyrrhetinic acid (2) derivatives towards new anti-inflammatory agents with improved properties regarding the natural product, it was decided, in 


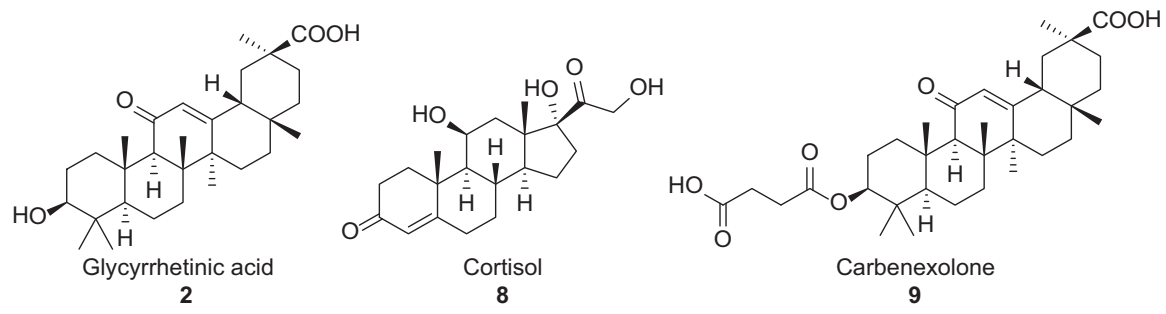

Fig. 4. Structures of Glycyrrhetinic acid (2), Cortisol (8), and Carbenexolone (9)

order to not extend this review size, to focus in the new reports of the antiinflammatory activity of $\mathbf{2}$ itself.

Puchner et al. tested the efficacy of $\mathbf{2}$ in a model of rheumatoid arthritis (hTNFtg mice) and found that the triterpene was not effective in this model when compared with the positive control (TNF inhibitors) [64].

Investigating the anti-inflammatory activity of $\mathbf{2}$ in HepG2 cells, Chen et al. reported that it suppresses TNF-induced inflammation in this cell culture by diminishing NF- $\kappa \mathrm{B}$ activation [65].

Wang et al. reported that using 2 in RAW264.7 macrophages induced with LPS did not alter cell viability and suppressed the activity of NF- $\kappa \mathrm{B}$ and PI3K, inhibiting the expression of several proinflammatory genes and attenuating the production of $\mathrm{NO}$, prostaglandin $\mathrm{E}_{2}\left(\mathrm{PGE}_{2}\right)$, and reactive oxygen species (ROS) [66].

Studying the proinflammatory cytokine effect of $\mathbf{1}$ and $\mathbf{2}$, Kao et al. reported that both compounds are capable of suppressing cytokine production but by different mechanisms: while 1 work via PI3K/Akt/GSK3 $\beta$ pathway, 2 acts by dissociating the glucocorticoid receptor (GR)-HSP90 complex [67].

\subsection{Other Activities}

Zhang et al. reported that 2 is a competitive Glyoxalase I inhibitor, with $K_{\mathrm{i}}=0.29 \mu \mathrm{M}$. The authors also studied the crystal structure of the complex Glyoxalase I-glycyrrhetinic acid, indicating that the carboxylic acid moiety of the triterpene is important for the inhibitor-enzyme interaction [68].

Inspired in previous reports regarding the chemoprotective effect of $\mathbf{1}$ and $\mathbf{2}, \mathrm{Wu}$ et al. reported that $\mathbf{2}$ can act as a chemoprotective agent against cisplatin-induced nephrotoxicity in BALB/c mice. This effect is, according to the authors, related to upregulation of $\mathrm{Nrf2}$ and downregulation of $\mathrm{Nf}-\kappa \mathrm{B}$ [69].

Kong et al. reported that $\mathbf{2}$ can act as a skin protector against $\mathrm{UV}$-induced photoaging in a mouse model. The authors also found that this protective effect is probably due to the anti-inflammatory and antioxidative properties of 2 [70].

Moon et al. investigated the effects of $\mathbf{2}$ in in vitro models of adipogenesis and found that the triterpene alters fat mass by affecting adipogenesis in maturing preadipocytes and lipolysis in matured adipocytes [71]. Also investigating the 
antiobesity properties of Liquorice, Park et al. reported that $\mathbf{2}$ suppresses the activation of cannabinoid type 1 receptor (CB1R) induced by the endogenous agonist anandamide [72].

While researching the effects of 2 in the hemostasis, Jiang et al. found that glycyrrhetinic acid is a Factor $\mathrm{Xa}(\mathrm{FXa})$ inhibitor, with in vitro $\mathrm{IC}_{50}=32.6 \pm 1.24 \mu \mathrm{M}$. The authors also tested the in vivo effect in rats using two protocols (tail bleeding and venous stasis models) and reported that $\mathbf{2}$ can increase prothrombin time and reduce thrombus weight (at a $50 \mathrm{mg} / \mathrm{kg}$ dose) when compared to the control group [73].

Hardy et al. reported that $\mathbf{2}$ was able to inhibit rotavirus replication in in vitro assays, while $\mathbf{1}$ was ineffective in this assay. The authors also reported that the antiviral activity of $\mathbf{2}$ occurs after the entry stage of the viral cycle [74].

\section{$4 \quad$ Concluding Remarks}

A great range of pharmacological and biological activities have been reported in the literature in the last 5 years, ranging from the exploration of known activities (such as the signaling pathways responsible for the anticancer and anti-inflammatory activity of $\mathbf{1}$ and 2), scope broadening (the investigation of the antibacterial and antiviral properties against new, unreported species of virus and bacteria) and also new, unreported activities (such as the antiparasitic activities, glyoxalase I, factor Xa, etc.). Several clinical trials regarding the anti-inflammatory and hepatoprotective activity of $\mathbf{1}$ were also discussed.

The wide range of reported activities of $\mathbf{1}$ and $\mathbf{2}$ can lead to a very important question: how safe it is the use of $\mathbf{1}$ as a sweetener? Glycyrrhizinic acid is currently considered "Generally recognized as safe" by the US Food and Drug Administration, and there is a guideline for maximum permitted levels of the saponin in several preparations [75]. Also, the European Commission report on Glycyrrhizin states that the saponin is safe for consumption with the warning that the maximum daily dose of 1 should not be higher than $100 \mathrm{mg}$ because of the glucocorticoid effects of glycyrrhetinic acid (2), given that the saponin is absorbed by the intestine as the aglycone, after hydrolysis of its carbohydrate moiety [2].

Regarding the reported properties for both products, most of the published articles explored in this chapter describe the in vitro or experimental in vivo activities, many of them in $\mu \mathrm{M}$ levels, which are considered too high to be useful as a drug [76] unless a very high dose is administered - way over the quantities permitted by the regulatory boards in both European Union [2] and USA [74]. Those activities should be regarded as potential ones, being useful as prototypes for the design and synthesis of new, improved compounds structurally related to $\mathbf{1}$ and/or $\mathbf{2}$.

Glycyrrhizinic acid can be, described, eventually, as more than a sweetener. Along with glycyrrhetinic acid, it can be considered as scaffold molecule for the design and development of new bioactive compounds. 
Acknowledgments This work is partly supported by grants from the Brazilian Government Science and Research Council (in Portuguese: Conselho Nacional de Pesquisa or CNPq) and the Rio de Janeiro State Research Foundation (Fundação de Apoio à Pesquisa do Estado do Rio de Janeiro or FAPERJ).

\section{References}

1. World Health Organization (2006) Safety evaluation of certain food additives. WHO Press, Geneva

2. European Comission Scientific Comiteee on Food (2003) Opinion of the scientific committee on food on glycyrrhizinic acid and its ammonium salt. European Commission. http://ec.europa. eu/food/safety/docs/fs-improv-flavourings-out186 en.pdf. Accessed 29 Jan 2016

3. Graebin CS, Verli H, Guimarães JA (2010) Glycyrrhizin and glycyrrhetic acid: scaffolds to promising new pharmacologically active compounds. J Braz Chem Soc 21:1595-1615

4. Ijeda K, Arase Y, Kobayashi M, Saitoh S, Someya T, Hosaka T, Sekazi H, Akuta N, Suzuki S, Suzuki F, Kumada H (2006) A long-term glycyrrhizin injection therapy reduces hepatocellular carcinogenesis rate in patients with interferon-resistant active chronic hepatitis $\mathrm{C}$ : a cohort study of 1249 patients. Dig Dis Sci 51:603-609

5. Glavac KN, Kreft S (2012) Excretion profile of glycyrrhizin metabolite in human urine. Food Chem 131:305-308

6. Baltina LA (2003) Chemical modification of glycyrrhizic acid as a route to new bioactive compounds for medicine. Curr Med Chem 10:155-171

7. Ming LJ, Yin ACY (2013) Therapeutic effects of glycyrrhizic acid. Nat Prod Comm 8:415-418

8. American Chemical Society (2016) SciFinder. http://scifinder.cas.org. Accessed 29 Jan 2016.

9. Wang H, Chu Z, Gao Y, Huang M (2015) Effect of glycyrrhizin on gastric cancer BGC-823 cell proliferation. Shijie Huaren Xiaohua Zazhi 23:2868-2873

10. Yang YA, Tang WJ, Zhang X, Yuan JW, Liu XH, Zhu HL (2014) Synthesis, molecular docking and biological evaluation of glycyrrhizin analogs as anticancer agents targeting EGFR. Molecules 19:6368-6381

11. Huang RY, Chu YL, Jiang ZB, Chen XM, Zhang X, Zeng X (2014) Glycyrrhizin suppresses lung adenocarcinoma cell growth through inhibition of thromboxane synthase. Cell Phys Biochem 33:375-388

12. Smolarczyk R, Cichon T, Matuszczak S, Mitrus I, Lesiak M, Kobusinska M, Kamysz W, Jarosz M, Sieron A, Szala S (2012) The role of glycyrrhizin, an inhibitor of HMGB1 protein, in anticancer therapy. Arch Immun Therap Exper 60:391-399

13. Wang W, Chen X, Zhang J, Zhao Y, Li S, Tan L, Gao J, Fang X, Luo A (2015) Glycyrrhizin attenuates isoflurane-induced cognitive deficits in neonatal rats via its anti-inflammatory activity. Neuroscience 316:328-336

14. Zhang J, Wu Y, Weng Z, Zhou T, Feng T, Lin Y (2014) Glycyrrhizin protects brain against ischemia-reperfusion injury in mice through HMGB1-TLR4-IL-17A signaling pathway. Brain Res 1582:176-186

15. Liu Z, Zhong J, Gao E, Yang H (2014) Effects of glycyrrhizin acid and licorice flavonoids on LPS-induced cytokines expression in macrophage. Zhongguo Zhongyao Zazhi 39:3841-3845

16. Fu Y, Zhou E, Wei Z, Song X, Liu Z, Wang T, Wang W, Zhang N, Liu G, Yang Z (2014) Glycyrrhizin inhibits lipopolysaccharide-induced inflammatory response by reducing TLR4 recruitment into lipid rafts in RAW264.7 cells. Biochimica Biophys Acta Gen Subjects 1840:1755-1764

17. Wang HL, Li YX, Niu YT, Zheng J, Wu J, Shi GJ, Ma L, Niu Y, Sun T, Yu JQ (2015) Observing anti-inflammatory and anti-nociceptive activities of glycyrrhizin through regulating COX-2 and pro-inflammatory cytokines expressions in mice. Inflammation 38:2269-2278

18. Chang CZ, Wu SC, Kwan AL (2015) Glycyrrhizin attenuates proinflammatory cytokines through a peroxisome proliferator-activated receptor- $\gamma$-dependent mechanism and experimental vasospasm in a rat model. J Vasc Res 52:12-21 
19. Chang CZ, Wu SC, Kwan AL (2014) Glycyrrhizin attenuates toll like receptor-2, -4 and experimental vasospasm in a rat model. J Immun Res 740549:1-9

20. Xiao J, Liu J, Zhang Q (2014) Influence of compound glycyrrhizin on the functions of Treg and TH17 cells in children with henochschonlein purpura. Qingdao Daxue Yixueyuan Xuebao 50:315-317

21. Zhou J, Ma N, Qie Y, Shen J (2014) Efficiency of compound glycyrrhizin in treatment of 42 cases with chronic urticaria. Zhongguo Yaoye 23:117

22. Luo X, Li X, Yang L, Wang X (2014) Efficacy of magnesium isoglycyrrhizinate in treatment of hepatitis E with severe jaundice. Linchuang Gandanbing Zazhi 30:537-539

23. Zhang XX, Wu J, Xu Y, Zou X, Hu SY (2014) Clinical observation of compound glycyrrhizin for the prevention of liver and hematological system toxicity after chemotherapy for digestive tract cancer. Zhongguo Yaofang 25:4364-4366

24. Hsiang CY, Lin LJ, Kao ST, Lo HY, Chou ST, Ho TY (2015) Glycyrrhizin, silymarin, and ursodeoxycholic acid regulate a common hepatoprotective pathway in HepG2 cells. Phytomedicine 22:768-777

25. Meng W, Yu LZ, Wang L (2013) Effects of compound glycyrrhizin on liver function in patients with alcoholic liver disease: a meta-analysis. Zhongguo Yaofang 24:1116-1118

26. Yin J, Feng H, Chen S, Yang L (2013) Effects of compound glycyrrhizin preparation on IL-17 and ANA in patients with chronic hepatitis B. Jiangsu Yiyao 39:1280-1281

27. Cai $\mathrm{L}$, Wu Y, Zhang J (2012) Comparison of the efficacy of two glycyrrhizin in treatment of chronic hepatitis B. Yaowu Liuxingbingxue Zazhi 21:160-161

28. Ding TP, Qu Y, Lu LG, Shi GL, Wang RF (2012) 18 $\alpha$-Glycyrrhizin ameliorates oxidative stress in rats with $\mathrm{CCl}_{4}$-induced liver fibrosis. Shijie Huaren Xiaohua Zazhi 20:1819-1823

29. Smith G, Stubbins MJ, Harries LW, Wolf CR (1999) Molecular genetics of the human cytochrome P450 monooxygenase superfamily. Xenobiotica 28:1129-1165

30. Wang YG, Zhou JM, Ma ZC, Li H, Liang QD, Tan HL, Xiao CR, Zhang BL, Gao Y (2012) Pregnane $X$ receptor mediated-transcription regulation of CYP3A by glycyrrhizin: a possible mechanism for its hepatoprotective property against lithocholic acid-induced injury. ChemicoBiol Interact 200:11-20

31. Chen Y, Wan X, Sun J, Wang W, Zhang S (2012) Hepato-protective activity of glycyrrhizin, liquiritin and isoliquiritigenin on HL-7702 cells injury induced by acetaminophen. Zhongguo Shiyan Fangjixue Zazhi 18:245-248

32. Gwak GY, Moon TG, Lee DH, Yoo BC (2012) Glycyrrhizin attenuates HMGB1-induced hepatocyte apoptosis by inhibiting the p38-dependent mitochondrial pathway. World J Gastr $18: 679-684$

33. Duan E, Wang D, Fang L, Ma J, Luo J, Chen H, Li K, Xiao S (2015) Suppression of porcine reproductive and respiratory syndrome virus proliferation by glycyrrhizin. Antivir Res 120:122-125

34. Li Z, Zhao Y, Lin W, Ye M, Ling X (2015) Rapid screening and identification of active ingredients in licorice extract interacting with V3 loop region of HIV-1 gp120 using ACE and CE-MS. J Pharm Biom Anal 111:28-35

35. Matsumoto Y, Matsuura T, Aoyagi H, Matsuda M, Hmwe SS, Date T, Watanabe N, Watashi K, Suzuki R, Ichinose S, Wake K, Suzuki T, Miyamura T, Wakita T, Aizaki H (2013) Antiviral activity of glycyrrhizin against hepatitis $\mathrm{C}$ virus in vitro. PLoS One 8:e68992

36. Ashfaq UA, Nawaz Z, Riazuddin S (2011) Glycyrrhizin as antiviral agent against Hepatitis C virus. J Trans Med 9:112

37. Xiao Q (2014) Zhou X (2014) Glycyrrhizin inhibits human neutrophil elastase-induced mucin 5 AC overproduction in human bronchial epithelial cells. Zhongnan Daxue Xuebao, Yixueban 39:252-257

38. Sil R, Sen S, Chakraborti AS (2015) Interaction of glycyrrhizin with human haemoglobin. Curr Sci 108:364-371

39. Francischetti IMB, Monteiro RQ, Guimarães JA (1997) Identification of glycyrrhizin as a thrombin inhibitor. Biochem Biophys Res Comm 9:259-263 
40. Mendes-Silva W, Assafim M, Ruta B, Monteiro RQ, Guimarães JA, Zingali RB (2003) Antithrombotic effect of glycyrrhizin, a plant-derived thrombin inhibitor. Thromb Res 112:93-98

41. Paula FT, Frauches PQ, Pedebos C, Berger M, Gnoatto SC, Gossmann G, Verli H, Guimarães JA, Graebin CS (2013) Improving the thrombin inhibitory activity of glycyrrhizin, a triterpenic saponin, through a molecular simplification of the carbohydrate moiety. Chem Biol Drug Design 82:756-760

42. Imai K, Takagi Y, Iwazaki A, Nakanishi K (2014) Radical scavenging ability of glycyrrhizin. Free Rad Antioxid 3:40-42

43. Csuk R (2014) Recent developments in the synthesis of antitumor-active glycyrrhetinic acid derivatives. Mini-Rev Org Chem 11:253-261

44. Kim H, Kim SY, Lee JH, Han Y (2011) Antitumor effect of 18ß-glycyrrhetinic acid against human tumor xenografts caused by A549 cancer cell. Yakhak Hoechi 55:39-44

45. Yamaguchi H, Yu T, Kidachi Y, Akitaya T, Yoshida K, Kamiie K, Noshita T, Umetsu H, Ryoyama K (2011) Selective toxicity of glycyrrhetinic acid against tumorigenic $\mathrm{r} / \mathrm{m}$ HM-SFME-1 cells is potentially attributed to downregulation of glutathione. Biochimie 93:1172-1178

46. Xu S, Zhou L, Liu Z, Chen B, Li Y (2011) Glycyrrhetinic acid induces apoptosis and alters survivin gene expression in human myeloma cell line U266. Zhongguo Shiyan Xueyexue Zazhi 19:652-655

47. Kowsalya R, Vishwanathan P, Manoharan S (2011) Chemopreventive potential of $18 \beta$-glycyrrhetinic acid: an active constituent of liquorice, in 7,12-dimethylbenz(a)anthracene induced hamster buccal pouch carcinogenesis. Pakistan J Biol Sci 14:619-626

48. Sharma G, Kar S, Palit S, Das PK (2012) 18ß-glycyrrhetinic acid (concur) induces apoptosis through modulation of Akt/FOXO3a/Bim pathway in human breast cancer MCF-7 cells. J Cell Phys 227:1923-1931

49. Kuang P, Zhao W, Su W, Zhang Z, Zhang L, Liu J, Ren G, Yin Z, Wang X (2013) $18 \beta$-glycyrrhetinic acid inhibits hepatocellular carcinoma development by reversing hepatic stellate cell-mediated immunosuppression in mice. Int J Cancer 132:1831-1841

50. Xie F, Cao Y, Liu GR, Yang XH, Dai Q, Chen W (2013) Mechanism underlying the inhibitory effect of glycyrrhetinic acid on epidermal growth factor-induced proliferation of $\mathrm{HaCaT}$ cells. Zhonghua Pifuke Zazhi 46:278-281

51. Li ZL, Sun D, Yang YC, Xu XL, Kong CZ (2012) $18 \beta$-glycyrrhetinic acid inhibits human $17 \beta$-hydroxysteroid dehydrogenase type III and induces cell apoptosis via activation of eIF2 $\alpha$ in prostate cancer. Zhongguo Yike Daxue Xuebao 41:882-885

52. Wang D, Wong HK, Feng YB, Zhang ZJ (2014) 18beta-Glycyrrhetinic acid induces apoptosis in pituitary adenoma cells via ROS/MAPKs-mediated pathway. J Neuro-Oncology 116:221-230

53. Huang RY, Chu YL, Huang QC, Chen XM, Jiang ZB, Zhang X, Zeng X (2014) $18 \beta$-Glycyrrhetinic acid suppresses cell proliferation through inhibiting thromboxane synthase in non-small cell lung cancer. PLoS One 9:e93690

54. Jayasooriya RGPT, Dilshara MG, Park SR, Choi YH, Hyun JW, Chang WY, Kim GY (2014) $18 \beta$-Glycyrrhetinic acid suppresses TNF- $\alpha$ induced matrix metalloproteinase- 9 and vascular endothelial growth factor by suppressing the Akt-dependent NF-kB pathway. Toxicol In Vitro 28:751-758

55. Mahmoud AM, Al Dera HS (2015) 18 $\beta$-glycyrrhetinic acid exerts protective effects against cyclophosphamide-induced hepatotoxicity: potential role of PPAR $\gamma$ and Nrf2 upregulation. Genes Nutr 10:1-13

56. Chen S, Zou L, Li L, Wu T (2013) The protective effect of glycyrrhetinic acid on carbon tetrachloride-induced chronic liver fibrosis in mice via upregulation of Nrf2. PLoS One 8:e53662

57. Hasan SK, Khan R, Ali N, Khan AQ, Rehman MU, Tahir M, Lateef A, Nafees S, Mehdi SJ, Rashid S, Shalid A, Sultana S (2015) 18- $\beta$ glycyrrhetinic acid alleviates 2-acetylaminofluoreneinduced hepatotoxicity in Wistar rats: role in hyperproliferation, inflammation and oxidative stress. Human Exp Toxicol 34:628-641 
58. Gupta P, Das PK, Ukil A (2015) Antileishmanial effect of $18 \beta$-glycyrrhetinic acid is mediated by toll-like receptor-dependent canonical and noncanonical p38 activation. Antimicrob Ag Chemother 59:2531-2539

59. Ukil A, Kar S, Srivastav S, Ghosh K, Das PK (2011) Curative effect of $18 \beta$-glycyrrhetinic acid in experimental visceral leishmaniasis depends on phosphatase-dependent modulation of cellular MAP kinases. PLoS One 6:e29062

60. Kalani K, Agarwal J, Alam S, Khan F, Pal A, Srivastava SK (2013) In silico and in vivo antimalarial studies of $18 \beta$ glycyrrhetinic acid from Glycyrrhiza glabra. PLoS One 8:e74761

61. Kalani K, Kushwaha V, Verma R, Murthy PK, Srivastava SK (2013) Glycyrrhetinic acid and its analogs: a new class of antifilarial agents. Bioorg Med Chem Lett 23:2566-2570

62. Long DR, Mead J, Hendricks JM, Hardy ME, Voyich JM (2013) 18 $\beta$-glycyrrhetinic acid inhibits methicillin-resistant Staphylococcus aureus survival and attenuates virulence gene expression. Antimicrob Ag Chemother 57:241-247

63. Kim SR, Jeon HJ, Park HJ, Kim MK, Choi WS, Jang HO, Bae SK, Jeong CH, Bae MK (2013) Glycyrrhetinic acid inhibits Porphyromonas gingivalis lipopolysaccharide-induced vascular permeability via the suppression of interleukin-8. Inflammation Res 62:145-154

64. Puchner A, Hayer S, Niederreiter B, Hladik A, Blueml S, Bonelli M, Scheinecker C, Smolen J, Redlich K (2012) Effects of $18 \beta$-glycyrrhetinic acid in hTNFtg mice - a model of rheumatoid arthritis. Wiener Klinische Wochenschrift 124:170-176

65. Chen HJ, Kang SP, Lee IJ, Lin YL (2014) Glycyrrhetinic acid suppressed NF-кB activation in TNF- $\alpha$-induced hepatocytes. J Agric Food Chem 62:618-625

66. Wang CY, Kao TC, Lo WH, Yen GC (2011) Glycyrrhizic acid and 18 $\beta$-glycyrrhetinic acid modulate lipopolysaccharide-induced inflammatory response by suppression of NF- $\kappa \mathrm{B}$ through PI3K p1108 and p110 $\gamma$ inhibitions. J Agric Food Chem 59:7726-7733

67. Kao TC, Shyu MH, Yen GC (2010) Glycyrrhizic acid and $18 \beta$-glycyrrhetinic acid inhibit inflammation via PI3K/Akt/GSK3 $\beta$ signaling and glucocorticoid receptor activation. J Agric Food Chem 58:8623-8629

68. Zhang H, Huang Q, Zhai J, Zhao Y, Zhang L, Chen Y, Zhang R, Li Q, Hu X (2015) Structural basis for 18- $\beta$-glycyrrhetinic acid as a novel non-GSH analog glyoxalase I inhibitor. Acta Pharmacol Sinica 36:1145-1150

69. Wu CH, Chen AZ, Yen GC (2015) Protective effects of glycyrrhizic acid and $18 \beta$-glycyrrhetinic acid against cisplatin-induced nephrotoxicity in BALB/c mice. J Agric Food Chem 63:1200-1209

70. Kong SZ, Chen HM, Yu XT, Zhang X, Feng XX, Kang XH, Li WJ, Huang N, Luo H, Su ZR (2015) The protective effect of $18 \beta$-glycyrrhetinic acid against UV irradiation induced photoaging in mice. Exp Geront 61:147-155

71. Moon MH, Jeong JK, Lee YJ, Seol JW, Ahn DC, Kim IS, Park SY (2012) 18 $\beta$-glycyrrhetinic acid inhibits adipogenic differentiation and stimulates lipolysis. Biochem Biophys Res Comm 420:805-810

72. Park M, Lee JH, Choi JK, Hong YD, Bae IH, Lim KM, Park YH, Ha H (2014) $18 \beta$-glycyrrhetinic acid attenuates anandamide-induced adiposity and high-fat diet induced obesity. Mol Nutr Food Res 58:1436-1446

73. Jiang L, Wang Q, Shen S, Xiao T, Li Y (2014) Discovery of glycyrrhetinic acid as an orally active, direct inhibitor of blood coagulation factor Xa. Thromb Res 133:501-506

74. Hardy ME, Hendricks JM, Paulson JM, Faunce NR (2012) 18 $\beta$-glycyrrhetinic acid inhibits rotavirus replication in culture. Virology $\mathrm{J}$ 9:96

75. Food and Drug Administration (2015) CFR - code of federal regulations title 21. US Food and Drug Administration. https://www.accessdata.fda.gov/scripts/cdrh/cfdocs/cfcfr/CFRSearch. $\mathrm{cfm} ? \mathrm{fr}=184.1408$. Accessed 29 Jan 2016

76. Dougall IG, Unitt J (2015) Evaluation of the biological activity of compounds: techniques and mechanism of action studies. In: Wermuth CG, Aldous D, Raboisson P, Rognan D (eds) The practice of medicinal chemistry, 4th edn. Elsevier, London 